\title{
Modelización de la función velocidad para las ondas $P$ a partir de las fases PKiKP y PKIKP
}

\author{
M. RAMÍREZ-NICOLÁS ${ }^{1,2}$ \\ ${ }^{1}$ Departamento de Matemática Aplicada. \\ Facultad de Informática, Universidad Complutense de Madrid. \\ ${ }^{2}$ Departamento de Física de la Tierra, Astronomía y astrofísica I \\ Facultad de Ciencias Físicas, Universidad Complutense de Madrid. \\ marramir@fis.ucm.es
}

Recibido: 26/04/2011

Aceptado: 02/06/2011

\section{Resumen}

La estructura interna de la Tierra está caracterizada por una serie de regiones, las cuales presentan diferentes propiedades físicas. Existen diferentes modelos como el IASPEI91 o el PREM, los cuales además de describir la estructura interna de la Tierra nos proporcionan los valores teóricos de las velocidades de propagación de las ondas que atraviesan cada una de estas regiones. Las ondas sísmicas que atraviesan el interior de la Tierra nos proporcionan información sobre la constitución de la misma. En este trabajo nos vamos a centrar en las ondas que atraviesan el núcleo interno PKIKP, y las que se reflejan en la superficie del mismo PKiKP. El objetivo de este trabajo es el de identificar los tiempos de llegada de las fases PKIKP y PKiKP en un sismograma, comparándolos son los teóricos propuestos por los modelos y obtener una expresión para la velocidad de propagación de las ondas sísmicas en la discontinuidad entre el núcleo externo y el interno a partir de la minimización de los residuos entre la llegada de la onda PKiKP y la PKIKP.Para dicho estudio se han seleccionado dos terremotos, uno ocurrido en Colombia el 26/04/1999 ( $\mathrm{Mw}=5.9)$ y el otro en Perú-Ecuador 16/11/2007 $(\mathrm{Mw}=6.8)$, y una serie de estaciones en un rango de distancias epicentrales comprendido entre los $130^{\circ}$ y $140^{\circ}$, ya que para distancias epicentrales menores de $130^{\circ}$ existen fenómenos de interferencia entre las fases PKiKP y PKIKP.

Palabras clave: Núcleo interno, IASPEI91, PREM, PKIKP, PKiKP.

\section{Modelling of P-waves velocity function from the PKiKP and PKIKP phases}

\begin{abstract}
The structure of the Earth is characterized by a number of regions which have different physical properties. For their study one uses models such PREM or IASPEI91. These models describe the internal structure of the Earth providing us the theoretical values of the velocity of the waves that pass through each of these regions. In this paper we focus on the waves that cross the inner core (PKIKP), and the reflected waves on the surface of the inner core (PKiKP). The aim of this study is to identify the PKiKP and PKIKP phases in a seismogram and compare them with the theoretical values obtained from the models. Another objective of this work is to propose an expression for the propagation velocity of seismic waves at the discontinuity between the outer and inner core from the minimization of waste of time (time difference between the arrival of the wave PKIKP and PKiKP). For this study we have selected two earthquakes, one occurred in Colombia, 04/26/1999 $(\mathrm{Mw}=5.9)$ and the other in Peru-Ecuador 16/11/2007 $(\mathrm{Mw}=6.8)$. We have analyzed only the seismograms from stations with
\end{abstract}


epicentral distances between $130^{\circ}$ and $140^{\circ}$, because of the interference phenomena between the PKIKP and the PKiKP for epicentral distances less than $130^{\circ}$.

Keywords: Inner core, IASPEI91, PREM, PKIKP, PKiKP.

Sumario: Introducción. 1. Metodología. 2. Observaciones. 2.1. Datos para el estudio de la PKiKP y la PKIKP. 3. Resultados. 3.1. Terremoto de Colombia de 1999. 3.2. Terremoto de Perú-Ecuador 2007. 3.3. Comparación de resultados entre los terremotos de Colombia y de Perú-Ecuador. 4. Conclusiones. 5. Agradecimientos. Referencias bibliográficas.

\section{Referencia normalizada}

Ramírez-Nicolás, M., (2011). Modelización de la función velocidad para las ondas P a partir de las fases PKiKP y PKIKP. Física de la Tierra Vol. 23 Núm. 1 (2011): 59-71

\section{Introducción}

Dos de los modelos más conocidos para describir las propiedades físicas de las diferentes regiones de la Tierra son el Preliminary Reference Earth Model (PREM) y el International Association of Seismology and Physics of the Earth's Interior (IASPEI91). El primero, propuesto por Dziewonski y Anderson (1981) analiza de forma teórica las velocidades sísmicas en el interior de la Tierra, usando para ello la densidad y la atenuación como funciones de la profundidad. El segundo, está construido a partir de los tiempos de llegada de las ondas sísmicas de los boletines del ISC (International Seismological Center), para una corteza continental por lo que introduce unos ligeros errores tanto para la corteza inferior como para el manto superior (Kennett y Engdahl, 1991). En los dos casos los modelos describen bien las capas más externas de la Tierra, sin embargo a medida que aumenta la profundidad disminuye la exactitud de los modelos, en especial para el núcleo externo y el núcleo interno.

Del conjunto de regiones que componen la Tierra, en este trabajo nos centraremos en las más profundas, el núcleo externo y el interno. Entre los $2900 \mathrm{~km}$ y los $5100 \mathrm{~km}$ de profundidad se extiende el núcleo externo (figura 1). Cuando las ondas atraviesan esta región pasan a denominarse PKP, transmitiéndose como ondas longitudinales. Una característica importante es que se encuentra en estado líquido (o de fusión) por lo que no existirá la propagación de ondas transversales.

El núcleo interno se extiende entre 5100 y $6700 \mathrm{~km}$ de profundidad, es de naturaleza sólida. Si las ondas que se transmiten por él lo hacen como ondas longitudinales alcanzan distancias epicentrales comprendidas entre los $143^{\circ}$ y $180^{\circ}$, y se denominan PKIKP (figura 1, Udías et al., 1997).

Entre el núcleo externo y el interno existe una zona de transición llamada zona $\mathrm{F}$, es una región poco conocida y con grandes inhomogeneidades fuertes contrastes físicos y variaciones en la composición.

Además de estas ondas mencionadas anteriormente existen otras denominadas PKiKP. Estas ondas se caracterizan porque se reflejan en la superficie del núcleo interno (figura 1). 
A lo largo de este trabajo se analizará el comportamiento de las ondas PKIKP y PKiKP descritas anteriormente en los primeros kilómetros del núcleo para obtener una expresión que ajuste mejor la velocidad de las ondas sísmicas que atraviesan esa región de la Tierra. Para ello se emplearan diferentes sismogramas correspondientes a telesismos.

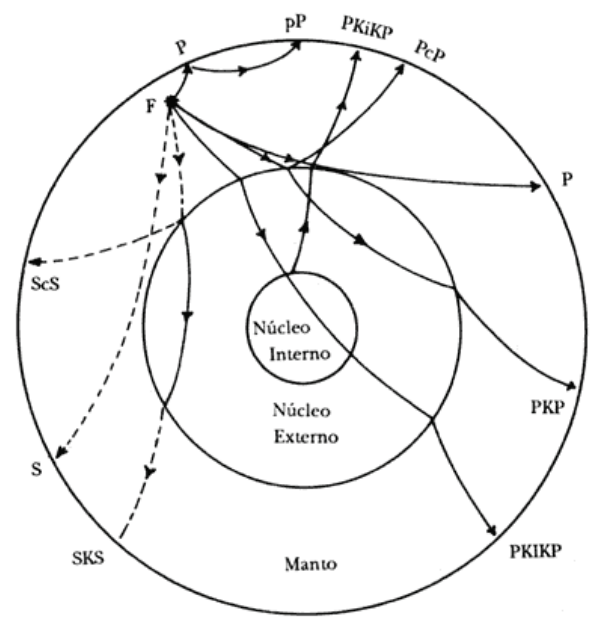

Fig. 1. Trayectorias de las ondas sísmicas en las capas internas de la Tierra. Las líneas continuas describen las trayectorias posibles para las ondas longitudinales (P) y las líneas discontinuas corresponden a las diferentes trayectorias descritas por las ondas transversales (S). http://bibliotecadigital.ilce.edu.mx/sites/ciencia/ volumen $3 /$ ciencia $3 / 113 / \mathrm{htm} / \mathrm{sec} 7 . \mathrm{htm}$

\section{Metodología}

La metodología empleada en este trabajo se basa en el estudio de las diferencias de tiempo o residuos calculados entre los tiempos de llegada a una misma estación de las ondas PKiKP y las PKIKP. Para ello determinaremos los tiempos de llegada de ambas ondas observados en estaciones con distancias epicentrales comprendidas en un rango de entre $130^{\circ}$ y $142^{\circ}$. El motivo por el cual se ha tomado este rango de distancias epicentrales se debe a que entre estos valores las ondas PKIKP apenas han penetrado en el núcleo interno, por lo que al calcular las diferencias de tiempo entre las fases PKiKP y PKIKP, afirmaremos que estas diferencias se van a deber a la estructura de aproximadamente los primeros $100 \mathrm{~km}$ del núcleo interno. Por otra parte, no se pueden tomar valores menores de $130^{\circ}$ porque para esas distancias epicentrales hay fenómenos de interferencia entre las ondas PKiKP y PKIKP, resultando difícilmente distinguibles.

Para identificar con mayor facilidad los tiempos de llegada de las fases se han tomado como referencia dichas fases en los sismogramas teóricos generados con el código DSM (Direct Solution Method) propuesto por N.Takeuchi basándose en el trabajo de Geller et al., 1995. De esta manera podremos identificar las fases PKIKP y PKiKP a partir de los tiempos teóricos de llegada (modelo IASP91 y PREM) y de las formas de onda generadas.

A partir de los tiempos de llegada observados para las fases PKIKP y PKiKP se han calculado los residuos a partir de la siguiente expresión. 


$$
(\Delta T)_{i}^{\text {OBSERVADO }}=t_{i}^{\text {PKiKP }}-t_{i}^{\text {PKIKP }} \quad i=1,2 \ldots N
$$

siendo $\mathrm{N}$ el número de estaciones que han registrado cada uno de los terremotos $\mathrm{y}$ $t_{i}$ el tiempo observado correspondiente a cada fase. Por otra parte se han calculado los valores de $(\Delta T)^{\text {TEORICO }}$ para estas fases, tanto para el modelo IASPEI como para el PREM, para ello se ha empleado un simulador de tiempos teóricos descrito en el código TTBOX y propuesto por Knapmeyer (2004). Comparando los residuos de $(\Delta T)^{\text {TEORICO }}$ y de $(\Delta T)^{\text {OBSERVADO }}$ se podrá concluir si las velocidades propuestas por los modelos PREM y IASPEI se ajustan a los valores observados, es decir, calcular el grado de ajuste entre los modelos teóricos que describen el comportamiento de la Tierra. A partir de esta premisa nos podemos encontrar en tres situaciones diferentes:

- $\mathrm{Si}(\Delta T)^{\text {TEORICO }}-(\Delta T)^{\text {OBSERVADO }}<0$ desviaciones por exceso de los modelos teóricos lo cual indica que las velocidades de propagación de las ondas en esta región son superiores a las propuestas por los modelos.

- $\mathrm{Si}(\Delta T)^{\text {TEORICO }}-(\Delta T)^{\text {OBSERVADO }}=0$ el ajuste entre los valores observados y los teóricos es total, lo cual indicaría un buen funcionamiento del modelo.

- $\mathrm{Si}(\Delta T)^{\text {TEORICO }}-(\Delta T)^{\text {OBSERVADO }}>0$ desviaciones respecto de los modelos teóricos son por defecto. En este caso las velocidades de propagación observadas serían inferiores de las propuestas por los modelos.

La principal hipótesis con la que se ha trabajado es que el recorrido de las fases PKIKP y PKiKP para estas distancias epicentrales es prácticamente el mismo y por tanto al utilizar las diferencias de tiempos entre estas fases, se eliminan en gran parte la influencia del modelo al calcular los tiempos teóricos (Niu et al., 2001).

Los modelos teóricos PREM y IASPEI que describen la estructura y composición interna de la Tierra, proponen una expresión cuadrática para las velocidades de propagación de las ondas longitudinales de la forma:

$$
V_{P}=a-b \cdot x^{2}
$$

En la expresión [3], $x=r / R$ representa el radio normalizado en el cual, $\mathrm{R}$ es el radio medio de la Tierra con un valor de $6371 \mathrm{~km} \mathrm{y} r$ es la variable profundidad que puede tomar valores comprendidos entre los $0 \mathrm{~km}$ y $6371 \mathrm{~km}$. 
Las diferencias entre ambos modelos (Ramírez, 2009) residen fundamentalmente en los términos cuadráticos, es decir, en los parámetros $b$, los cuales hacen referencia a la relación que existe entre la expresión de la velocidad y la profundidad (presión). Las diferencias entre los modelos se hacen más evidentes en el punto de intersección entre ambos (en torno a los $602 \mathrm{~km}$ desde el centro de la Tierra, es decir, en el Núcleo interno). Existen otros modelos que describen el Núcleo interno de la Tierra, como propuesto en el trabajo de Belonoshko et al. (2007), en el cual asume un núcleo compuesto íntegramente por hierro (Fe-bcc).

Uno de los objetivos de este trabajo es el de estudiar es si existe alguna relación entre la pendiente de esta curva calculada (término b) y la obtenida por los modelos IASP91 y PREM. Para ello lo que se ha hecho es calcular la variación de la velocidad de las ondas $\mathrm{P}$ en función del radio normalizado, y que vienen dadas por la ecuación 3.

$$
\begin{aligned}
& \left(\frac{d V_{P}}{d x}\right)_{\text {PREM }}=-12.728 \cdot x \\
& \left(\frac{d V_{P}}{d x}\right)_{\text {IASPEI } 91}=-8.194 \cdot x \\
& \left(\frac{d V_{P}}{d x}\right)_{F e(b c c)}=-16.123 \cdot x
\end{aligned}
$$

En la figura 2 se han representado las ecuaciones [3], para los tres modelos con los he se ha trabajado, el IASPEI91, el PREM y el Fe-bcc. En ella se observa que existe una gran divergencia en el comportamiento entre los modelos teóricos PREM i IASP91 (rojo y verde en la figura) y el calculado a partir de una composición puramente de hierro, para los primeros $100 \mathrm{~km}$ del núcleo interno (en negro en la figura 2).

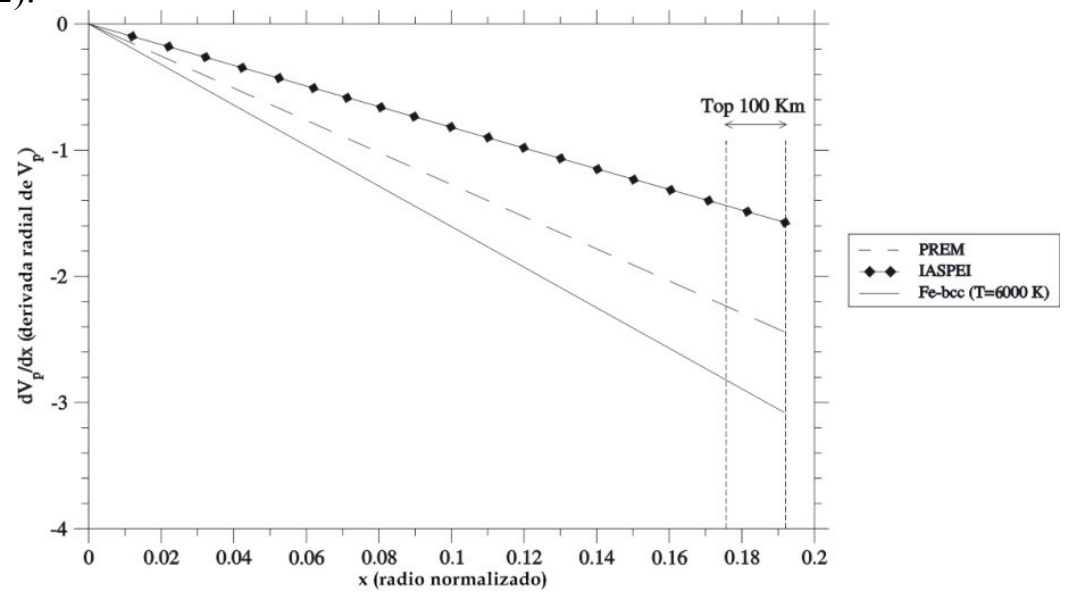

Fig. 2. Derivada radial de la $V_{\mathrm{P}}$ frente al radio normalizado para el núcleo interno para diferentes modelos de Tierra. 
A partir de estos resultados comprobamos como existe una mayor variación en el modelo propuesto (Fe bcc), respecto de los modelos teóricos PREM e IASP91.

\section{Observaciones}

\subsection{Datos para el estudio de la PKiKP y la PKIKP.}

Para realizar este estudio se han seleccionado dos terremotos, uno que ocurrió en Colombia el 26/04/1999 $(\mathrm{Mw}=5.9)$ y el otro en Perú-Ecuador del 16/11/2007 (Mw $=6.8)$ (tabla 1).

Tabla 1. Parámetros focales de los terremotos estudiados (http://www.globalcmt.org/CMTsearch.html).

\begin{tabular}{|c|c|c|c|c|c|c|}
\hline Referencia & Fecha & $\begin{array}{c}\text { Hora } \\
(\mathrm{GMT})\end{array}$ & $\begin{array}{c}\text { Latitud } \\
\left({ }^{\circ}\right)\end{array}$ & $\begin{array}{c}\text { Longitud } \\
\left({ }^{\mathbf{o}}\right)\end{array}$ & $\begin{array}{c}\text { Profundidad } \\
(\mathrm{km})\end{array}$ & $\mathrm{Mw}$ \\
\hline Colombia & $26 / 04 / 1999$ & $18: 17: 30.10$ & -1.645 & -77.785 & 164 & 5.9 \\
\hline Perú & $16 / 11 / 2007$ & $03: 13: 04.90$ & -2.500 & -78.000 & 114 & 6.8 \\
\hline
\end{tabular}

El primero de estos sismos ya fue estudiado por Niu et al. (2001). El objetivo de estudiarlo en este trabajo es comprobar que la metodología que se ha utilizado para la identificación de las fases PKIKP y PKiKP es la correcta, comparando los resultados de este trabajo con el de los citados autores. Una vez comprobada que la metodología desarrollada es válida se aplicará al sismo de Perú-Ecuador de 2007. Para estos sismos se han seleccionado aquellas estaciones que registraran dichos sismos en el $J$-Array (Jarray Group, 1993). El motivo de la selección de este array (o grupo de estaciones) es que está en un rango de distancias epicentrales comprendidas entre los $130^{\circ}$ y los $140^{\circ}$ y el bajo nivel de ruido en los registros, lo cual va a favorecer que las formas de onda de los sismogramas sean muy similares y la identificación de las fases sea más sencilla. Los registros empleados corresponden a sismogramas digitales de banda ancha del canal BHZ. Los sismogramas se obtuvieron de la base de datos japonesa, en su dirección en internet (http://jarray.eri.utokyo.ac.jp/).

Se seleccionaron para el terremoto de Colombia un total de 134 registros de los cuales tan sólo pudieron utilizarse 56 de ellos, y descartando el resto, bien por el alto contenido en ruido de los mismos o por la falta de precisión a la hora de identificar las fases. De igual forma para el sismo de Perú-Ecuador, de los 45 registros seleccionados, sólo pudieron utilizarse 20 de ellos. El procesado de los registros en formato SAC (Seismic Analysis Code), (Goldstein et al., 2003) se realizó convirtiéndolos en desplazamientos para posteriormente filtrarlos con el objetivo de simular un instrumento de periodo corto WWSSN. 


\section{Resultados}

\subsection{Terremoto de Colombia de 1999}

Las estaciones seleccionadas corresponden a trayectorias de rayos que recorren el hemisferio oeste de la Tierra por tanto, el análisis de las fases PKIKP y PKiKP nos dará información sobre la estructura interna de esta zona. A partir de los sismogramas seleccionados se han identificado y determinado los tiempos observados para las fases PKiKP y PKIKP para los dos terremotos mencionados anteriormente usando para ello las formas de onda teóricas generadas por el código DSMTI (Niu et al., 2001).

En la figura 3 se han representado los 37 sismogramas BHZ según lo descrito anteriormente en un rango de distancias epicentrales comprendido entre los $131^{\circ} \mathrm{y}$ los $141^{\circ}$. Para cada uno de estos se han tomado los tiempos de llegada de las fases PKiKP y PKIKP basándonos en la forma de las ondas y en los tiempos de llegada teóricos.

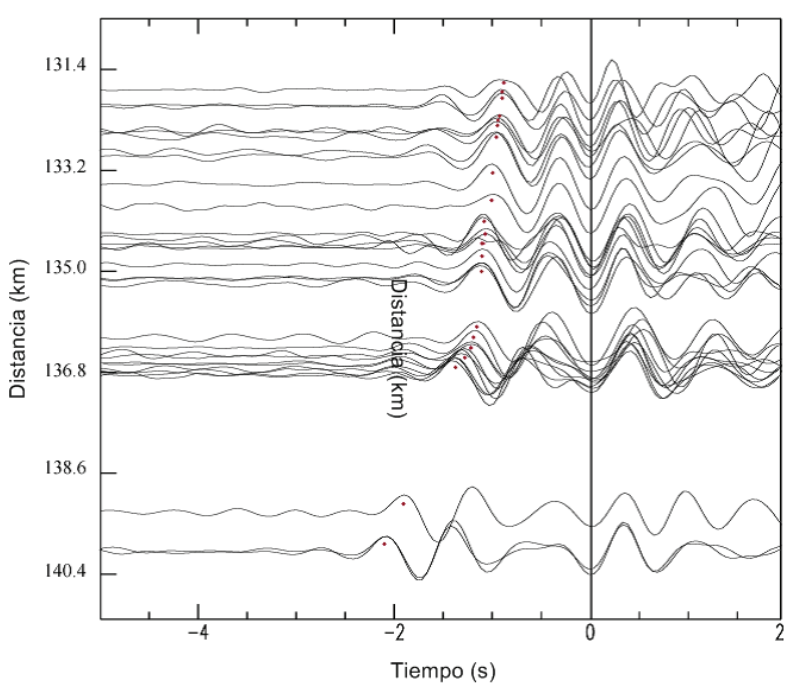

Fig. 3. Fases obtenidas experimentalmente, la llegada de la onda PKIKP (círculos) y PKiKP (línea vertical).

En dicha figura 3 se muestran las fases PKiKP y PKIKP perfectamente identificadas, dejando la evidencia de que a medida que aumenta la distancia epicentral también aumenta la diferencia entre los tiempos de llegada de las fases con las que estamos trabajando. Cada una de estas fases se han obtenido a partir de la comparación de las formas de onda de los sismogramas con las fases generadas de forma teórica con el código DSMTI (Niu et al., 2001).

De todos los valores calculados para los residuos (figura 4) se observa que el modelo que mejor ajusta es el PREM, ya que los valores de los residuos son muy próximos a cero, con valores comprendidos entre los $-0.572 \mathrm{~s}$ y los $0.350 \mathrm{~s}$. Mientras 
para el modelo IASP91 estos valores en general son superiores, variando entre $0.011 \mathrm{~s}$ y $0.922 \mathrm{~s}$, indicando un peor ajuste.

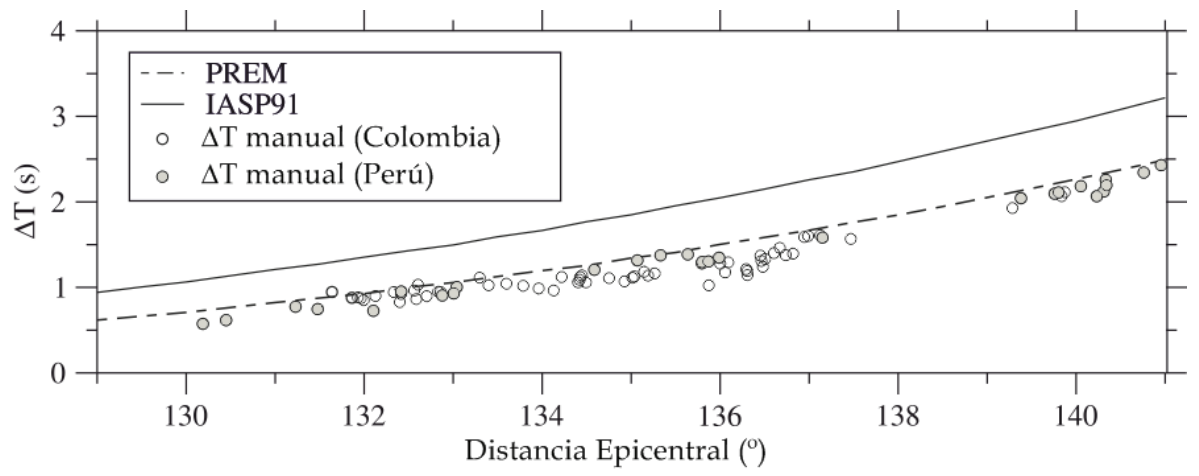

Fig. 4. Residuos PKIKP-PKiKP frente a distancia epicentral. La línea oscura corresponde a los valores propuestos por el modelo PREM, la línea clara por el IASPEI91, los círculos oscuros son residuos observados para el sismo de Colombia y los círculos claros para el de Perú-Ecuador.

\subsection{Terremoto de Perú-Ecuador 2007.}

Para este segundo terremoto se han seleccionado 20 registros BHZ, de estaciones comprendidas a distancias epicentrales entre los $130^{\circ}$ y los $140^{\circ}$. Los registros de velocidades en formato $\mathrm{SAC}$ se ha deconvolucionado con el instrumento y vuelto a convolucionar para poder simular como en el caso anterior un registro WWSSN de periodo corto. En la figura 5 se muestran la llegada de cada fase PKiKP y PKIKP para cada uno de los 20 sismogramas con los que se ha trabajado.

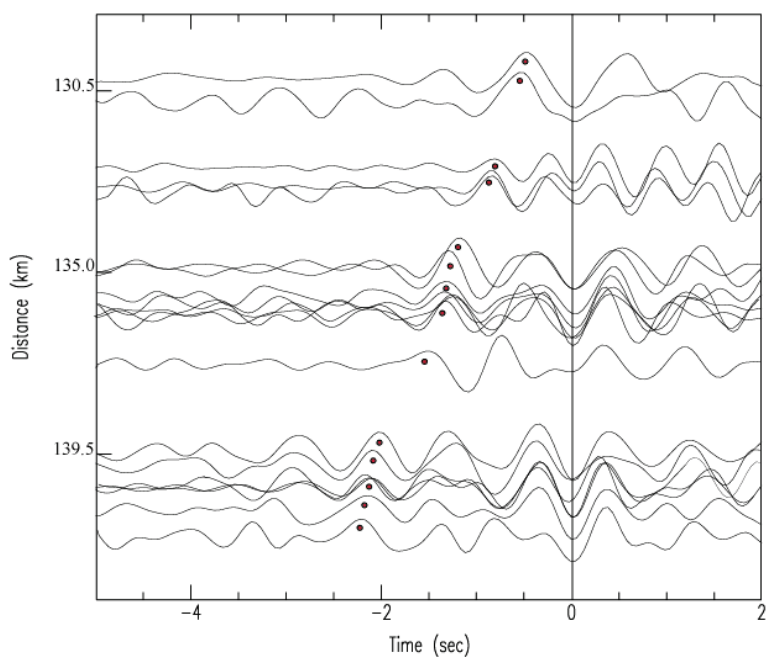


Fig. 5. Fases para el sismo de Perú- Ecuador. La llegada de la onda PKIKP (círculos) y PKiKP (línea vertical).

A pesar de que para estas estaciones los sismogramas contienen una mayor cantidad de ruido, se han podido identificar la llegada de la fase PKiKP y PKIKP mediante las formas de onda. Se han calculado los residuos (figura 4) entre estas fases comprobando a partir de estos, que los valores menores vuelven a corresponderse con los del modelo PREM. En esta ocasión los valores de los residuos son todavía más pequeños que en el caso del sismo de Colombia, con valores comprendidos para el caso del modelo PREM entre los $0.020 \mathrm{~s}$ y los $0.307 \mathrm{~s}$, mientras que para el modelo IASP91, a pesar de tener valores inferiores a los del caso anterior, siguen siendo mayores y están comprendidos entre $0.496 \mathrm{~s}$ y $1.055 \mathrm{~s}$.

\subsection{Comparación de resultados entre los terremotos de Colombia y de Perú- Ecuador.}

Si comparamos los valores de los residuos observados y de los residuos teóricos, tanto los correspondientes al modelo IASP91 como al PREM, para los dos terremotos analizados (figura 4), comprobamos que los observados son menores que los teóricos. Comparando en conjunto todos los $\Delta T$ observados para ambos terremotos, de un total de 57 valores, el $95 \%$ de los mismos son inferiores a los propuestos por el modelo PREM y el 98\% para el modelo IASPEI. Lo cual nos indica una sobrestimación en los tiempos de llegada de las fases PKiKP y PKIKP tanto en el modelo IASP91 como en el PREM.

Por otra parte comprobamos como las diferencias entre los valores teóricos y los observados son relativamente pequeños, diferenciándose respecto del modelo PREM en torno a 0.300 s (obteniendo generalmente valores más pequeños).

A la vista de lo observado en la figura 4, se puede concluir que el modelo que más se ajusta a nuestros valores en ambos casos resulta ser el modelo PREM. En un principio podría resultar sorprendente, ya que el modelo IASP91 es el más actual (por lo que debería ajustar mejor), pero no debemos olvidar que este modelo se generó a partir de una gran fuente de datos de sismos que ocurrieron en las regiones más superficiales de la Tierra. Por este motivo, al estudiar el núcleo interno no ofrece un buen ajuste.

El hecho de que los valores de los residuos observados sean menores que los correspondientes a los residuos de los modelos IASPEI91 y PREM, indica que las velocidades de propagación en los primeros $100 \mathrm{~km}$ del núcleo interno, son superiores a las ya estimadas. Por ello resulta de gran interés proponer una nueva expresión para la función de $\mathrm{V}_{\mathrm{P}}$, con el objetivo de poner encontrar una expresión que ajuste mejor los residuos observados. Para poder obtener esta expresión se ha optimizado el parámetro $b$ de la ecuación [2] de tal forma que se ha ido proponiendo valores hasta conseguir valores nulos para los residuos. Los resultados para ambos terremotos se muestran en las figura 6 y figura 7. 


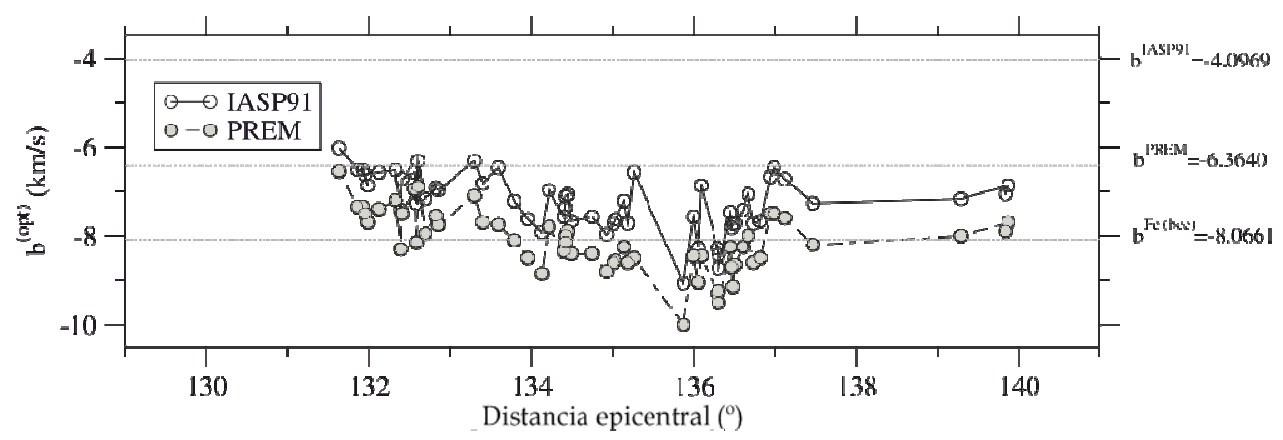

Fig. 6. Optimización del término b para la función de distribución de velocidades de las ondas longitudinales para el terremoto de Colombia (1999) para los modelos IASPEI91 (arriba) y el PREM (abajo).

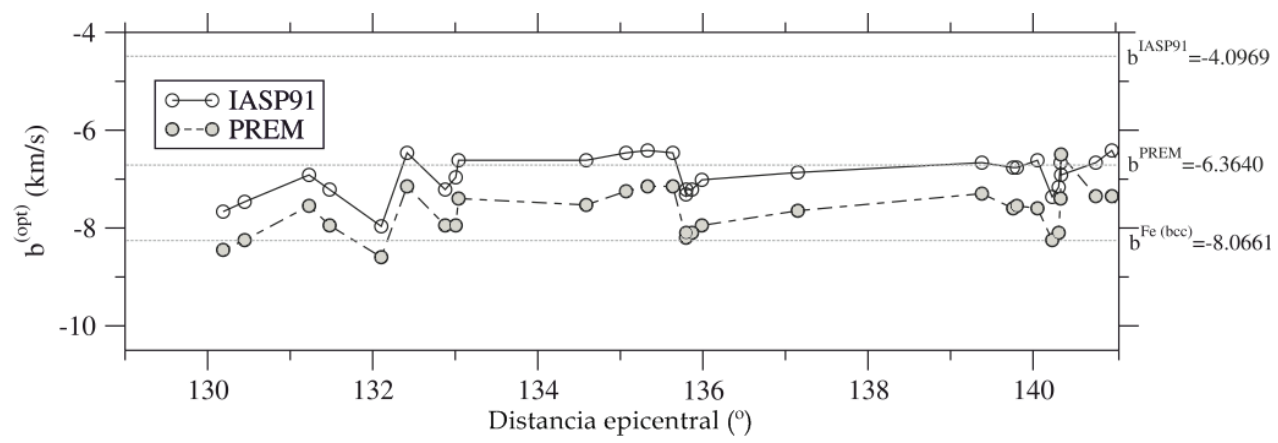

Fig. 7. Optimización del término b para la función de distribución de velocidades de las ondas longitudinales para el terremoto de Perú-Ecuador (2007) para los modelos IASPEI91 (arriba) y el PREM (abajo).

En ambos casos se puede observar como para mejorar el ajuste de nuestros valores observados a una función de distribución de velocidades del tipo $V_{P}=a-b \cdot x^{2}$, basta con que estos tengan un valor superior (en valor absoluto) al propuesto por los modelos IASP91 y PREM.

$\mathrm{Si}$ representamos las pendientes correspondientes a cada modelo teórico (IASP91, PREM y Fe bcc) y las relativas a los nuevos valores de b promediados para cada uno de los terremotos comprobamos como la pendiente de estos últimos se ajusta mucho mejor al modelo (Fe bcc) propuesto, que a los modelos IASPEI91 y PREM (figuras 8 y 9). 


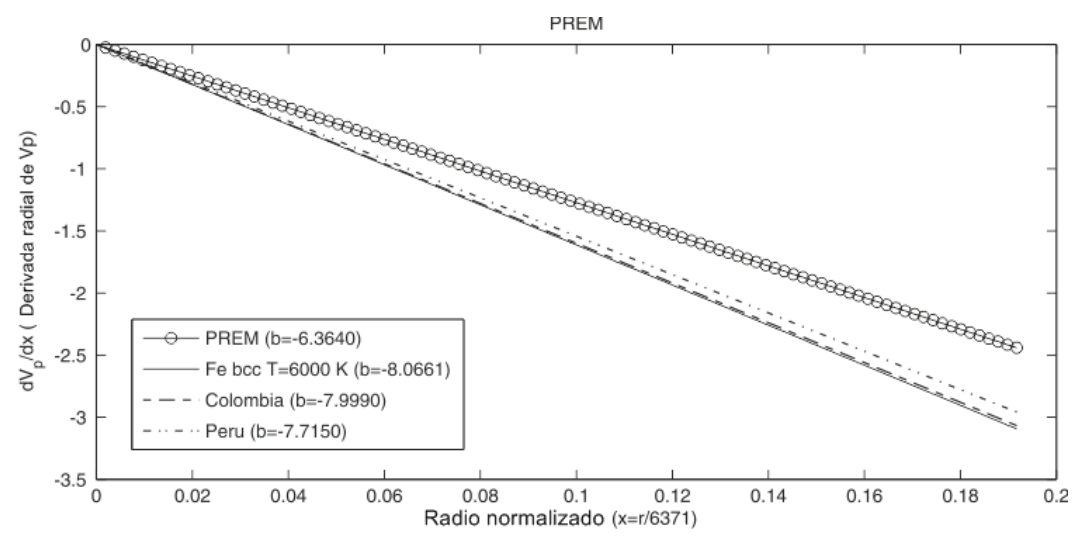

Fig. 8. Variación de la velocidad respecto del radio normalizado para el modelo PREM (círculos), el modelo Fe bcc (línea de puntos) y para los terremotos estudiados de Colombia (línea negra) y Perú- Ecuador (línea discontinua).

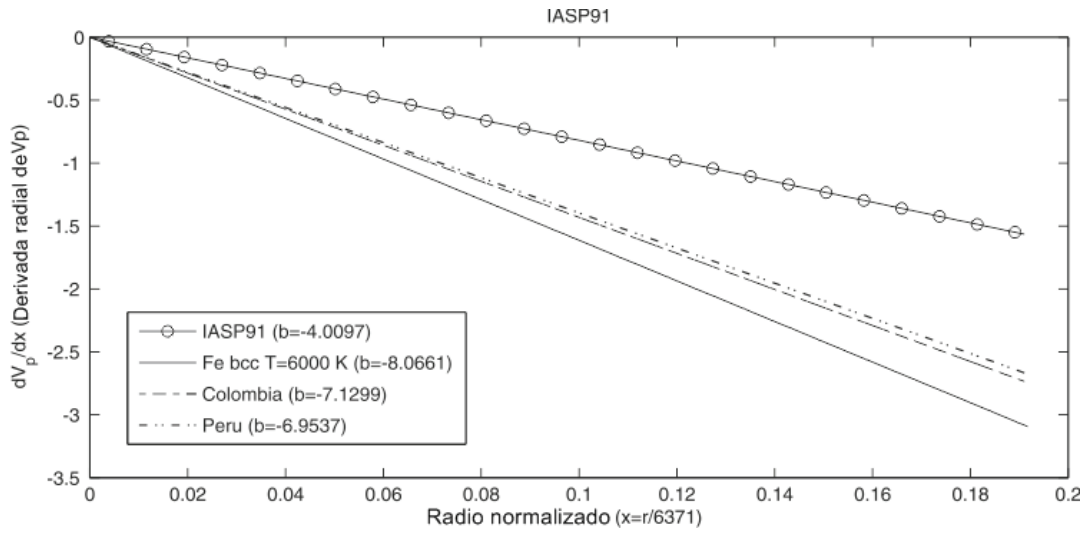

Fig. 9. Variación de la velocidad respecto del radio normalizado para el modelo IASP91 (línea con círculos), el modelo Fe bcc (línea de puntos) y para los terremotos estudiados de Colombia (línea negra) y Perú-Ecuador (línea discontinua).

A la vista de estos resultados se puede concluir que el modelo composicional propuesto a partir de Fe puro con estructura cúbica centrada en el cuerpo (bcc), representa un modelo químico-físico que se ajusta bastante bien a los valores experimentales, que representan el núcleo interno de la Tierra (Mattesini et al., 2010). Las ligeras diferencias existentes se podrían ver solventadas mediante el perfeccionamiento del modelo, es decir teniendo en cuenta pequeñas variaciones asociadas a la composición, en la que se introducirían algunos metales ligeros como $\mathrm{Si}, \mathrm{S}, \mathrm{O}, \mathrm{C}$ etc, que deberían refinar el comportamiento de estas funciones de distribución de velocidades (Mattesini et al., 2008). 


\section{Conclusiones}

De los resultados obtenidos a partir de los terremotos de Colombia de 1999 y PerúEcuador del 2007 podemos concluir:

La primera parte de este trabajo se ha centrado en el análisis y reproducción de los resultados obtenidos por Niu et al. (2001), con el objetivo de poder generalizarlos. Gracias a este trabajo se ha podido concluir la determinación de los tiempos de llegada de las fases PKIKP y PKiKP se convierte en una tarea muy complicada si no se realiza con la ayuda de sismogramas sintéticos. En este caso gracias a los sismogramas obtenidos mediante la utilización del código DMS propuesto por Takeuchi a partir de los trabajos de Geller (1995), y a partir de la identificación de la fase PKIKP, se han podido identificar la llegada de la onda PKiKP.

Por otra parte, una vez se pudieron determinar de forma experimental los valores de los tiempos de llegada de las fases PKIKP y PKiKP fue posible calcular el residuo entre estas dos fases. A la vista de los resultados obtenidos en la figura 6 , puede concluirse que, en general, todos los residuos observados tienen valores inferiores a los propuestos por los modelos IASP91 y PREM. Sin embargo si resultara necesario quedarse con alguna aproximación, se podría afirmar que con los valores observados, el modelo que mejor se ajusta resulta ser el PREM.

A pesar de estos resultados, resulta importante destacar que podría ser conveniente la mejora de este modelo teórico al aplicarse en los primeros $100 \mathrm{~km}$ del núcleo interno, ya que a la vista de los resultados las velocidades de propagación de estas ondas son superiores a las teóricas.

Por este motivo se podría encontrar una expresión que se ajuste mejor a los valores experimentales. Para conseguirlo bastaría con modificar el valor del parámetro $b$ de la misma, el cual debería ser en valor absoluto mayor que lo que proponen los modelos teóricos.

\section{Agradecimientos}

Parte de este trabajo se ha subvencionado con fondos de los proyectos los proyectos CGL 2008-00891 y GR58/08-910399. Además quisiera mostrar mi agradecimiento al proyecto AYA2009-14212-C05-05.

\section{Referencias bibliográficas}

BELONOSHKO, A. B., SKORODUMOVA, N. V., DAVIS, S., OSIPTSOV, A. N., ROSENGREN, A., \& JOHANSSON, B. (2007). Origin of the Low Rigidity of the Earth's Inner Core, Science, 316, 1603-1605.

doi: 10.1126/science.1141374; http://dx.doi.org/10.1126/science.1141374

DZIEWONSKY, A. M., \& ANDERSON, D.L. (1981). Preliminary reference Earth model, Physics of the Earth and Planetary Interiors, 25, 297-356.

doi: 10.1016/0031-9201(81)90046-7;

http://dx.doi.org/10.1016/0031-9201(81)90046-7 
GELLER, R, J., \& TAKEUCHI, N. (1995). A new method for computing highly accurate DSM synthetic seismograms, Geophysical Journal International, 123, 449-470. doi:10.1111/j.1365-246X.1995.tb06865.x; http://dx.doi.org/10.1111/j.1365-246X.1995.tb06865.x

GOLDSTEIN, P., DODGE, P., FIRPO, M., Y MINNER, L. (2003). “SAC2000: Signal processing and analysis tools for seismologists and engineers" Invited contribution to "the IASPEI International Handbook of Earthquake and Engineering Seismology" Edited by WHK Lee, H.Kanamori, P.C. Jennings and C.Kisslinger. Academic Press, London, 25.

doi:10.1016/S0074-6142(03)80284-X;

http://dx.doi.org/10.1016/S0074-6142(03)80284-X

J-ARRAY GROUP (1993). The J-Array Program: system and present status. Journal of Geomagnetism and Geoelectricity, 45, 1265-1274.

JEFFREYS, H. (1939). The times of P, S and SKS and the velocities of P and S. Mon. Nat. Roy. Astr. Soc. Geophys. Suppl, 4, 498-533.

KENNETT, B. L. N. (2005). Seismological tables AK135, Research School of Earthscience, The Australian National University.

KENNETT, B.L.N., \& ENGDAHL, E.R. (1991). Traveltime for global earthquake location and phase identification, J. Geophys. Int., 105, 429-465.

doi: 10.1111/j.1365246X.1991.tb06724.x;

http://dx.doi.org/10.1111/j.1365246X.1991.tb06724.x

KNAPMEYER, M. (2004). A Matlab Toolbox for the computation of 10 telesismic travel times, Seismological Research Letters, 75, 726.

doi: $10.1785 / \mathrm{gssrl} .75 .6 .726$;

http://dx.doi.org/10.1785/gssrl.75.6.726

MATTESINI, M., BUFORN, E., UDIAS, A., VITOS, L \& AHUJA, R. (2008). An ab initio study of S-substituted iron-nickel-silicon alloy at the Earth's inner core pressure, International Journal of High Pressure Research., 28, 437-441. doi:10.1080/08957950802497323; http://dx.doi.org/10.1080/08957950802497323

MATTESINI, M., BELONOSHKO, A. B., BUFORN, E., RAMÍREZ, M., SIMAK, S. I., UDÍAS, A., MAO, H-K., \& AHUJA, R. (2010). Hemispherical anisotropic patterns of the Earth's inner core, Proceedings of the National Academy of Sciences (PNAS), 107, 21, 9477-9916.

doi: 10.1073/pnas.1004856107;

http://dx.doi.org/10.1073/pnas.1004856107

NIU, F., \& LLANXING, W. (2001). Hemispherical variations in the seismic velocity at the top of the inner core, Nature, 410, 1081-1084.

doi: $10.1038 / 35074073$;

http://dx.doi.org/10.1038/35074073

RAMIREZ, M. (2009). Modelización de la función velocidad para las ondas P a partir de las fases PKIKP y PKiKP. Trabajo de Investigación del Máster en Geofísica y Meteorología. Departamento de Física de la Tierra, Astronomía y Astrofísica I. Universidad Complutense de Madrid.

UDÍAS, A., \& MEZCUA, J. (1997) Fundamentos de geofísica. Alianza Editorial, Madrid, $476 \mathrm{pp}$. 